\title{
利用瞬态苂光技术研究单重态激子裂变的分子间距 依赖关系
}

任晓伟，周亮，田晓语，张勇*

西南大学物理科学与技术学院, 重庆 400715

* 联系人, E-mail: yzh6127@swu.edu.cn

2015-10-21 收稿, 2015-12-03 修回, 2015-12-04 接受, 2016-04-19 网络版发表

国家自然科学基金(61475126)资助

摘要在红荧烯掺杂的有机薄膜中，通过控制红荧烯的掺杂浓度来改变相邻红荧烯分子间的平均间距，进而调 节相邻红荧烯分子间发生单重态激子裂变过程的速率. 实验上，利用瞬态荧光技术测量了不同掺杂样品光致发光 的瞬态衰减曲线. 理论上，基于“ $\mathrm{S}_{1}+\mathrm{S}_{0} \leftrightarrow{ }^{1}(\mathrm{TT})_{i} \leftrightarrow \mathrm{T}_{1}+\mathrm{T}_{1}$ ”三状态反应模型，采用耦合的速率方程组描述了红荧烯分 子间发生激子裂变的动力学过程, 并通过速率方程组的迭代运算拟合了实验测得的薄膜光致发光的瞬态衰减曲 线. 数据分析表明, 随着红荧烯分子平均间距的增大, $\mathrm{S}_{1}+\mathrm{S}_{0} \rightarrow^{1}(\mathrm{TT})$ 过程的速率呈指数下降规律, 这与有机材料中 常见的Dexter能量转移过程非常相似. 由于这两种过程都可以通过 “双电子转移”模型来理解，函数关系上的相似 性实际上是对激子裂变的双电子转移模型的验证, 这对于澄清激子裂变过程的物理机制具有重要价值.

关键词红荧烯, 光致发光, 激子裂变, 电子转移

单重态激子的裂变(singlet exciton fission, SF或 激子裂变), 是指在有机材料中由光或电激发产生一 个具有较高能量的单重态激子 $\mathrm{S}_{1}$, 然后 $\mathrm{S}_{1}$ 态激子把其 能量的一半转移给另一个处于基态的分子 $\mathrm{S}_{0}$, 随后 $\mathrm{S}_{1}$ 态激子和 $\mathrm{S}_{0}$ 态分子快速转变为两个具有较低能量的 三重态激子 $\mathrm{T}_{1}{ }^{[1,2]}$. 一般情况下, 激子裂变的动力学 过程可以简单地表示如下 ${ }^{[2]}$ :

$$
\mathrm{S}_{1}+\mathrm{S}_{0} \underset{k_{2}}{\stackrel{k_{-2}}{\rightleftharpoons}}(\mathrm{TT})_{i} \underset{k_{1}}{\stackrel{k_{-1}}{\rightleftharpoons}} \mathrm{T}_{1}+\mathrm{T}_{1}
$$

其中的 ${ }^{1}(\mathrm{TT})_{i}$ 态, 是由两个 $\mathrm{T}_{1}$ 态激子耦合形成的中间 过渡态, 而 $k_{1}, k_{-1}, k_{2}, k_{-2}$ 是不同状态间相互转化的速 率. 在过去几年里, 激子裂变过程和具有激子裂变特 性的有机材料受到研究者的广泛关注, 人们尝试将 能够发生激子裂变过程的有机分子作为一种新型的 敏化剂, 用来提高光伏器件的量子效率 ${ }^{[3 \sim 5]}$. 在器件
吸收 1 个光子后通过激子裂变产生 2 个 $\mathrm{T}_{1}$ 态激子, 若 这 2 个 $\mathrm{T}_{1}$ 态激子都解离成自由载流子, 则器件的量子 效率将大幅度提高. 迄今已经在多种有机分子中观 察到了激子裂变过程, 激子裂变的基本条件是 $E\left(\mathrm{~S}_{1}\right) \approx$ $2 E\left(\mathrm{~T}_{1}\right)^{[2]}$, 其中 $E\left(\mathrm{~S}_{1}\right)$ 和 $E\left(\mathrm{~T}_{1}\right)$ 分别为 $\mathrm{S}_{1}$ 态和 $\mathrm{T}_{1}$ 态激子的 能量. 在红苂烯中, $E\left(\mathrm{~S}_{1}\right)=2.23 \mathrm{eV}$, 而 $E\left(\mathrm{~T}_{1}\right)=1.14 \mathrm{eV}$, 满足 $E\left(\mathrm{~S}_{1}\right) \approx 2 E\left(\mathrm{~T}_{1}\right)$ 的条件, 因此在红苂烯分子间可发 生快速的激子裂变过程 ${ }^{[6-9]}$. 此外, 激子裂变过程是 可逆的, 其逆过程即所谓的三重态激子的聚变(triplet exciton fusion, TF或激子聚变 $)^{[2]}$, 通常这两种过程是 共存的.

目前, 人们对于激子裂变过程的物理机制还没 有一致的看法, 对于从 $\mathrm{S}_{1}+\mathrm{S}_{0}$ 态向 ${ }^{1}(\mathrm{TT})_{i}$ 态的转化过 程, 大多从两个角度来考虑. 一种是从“双电子转移” 的角度来分析 ${ }^{[2,10 ~ 12]}$, 即将激子裂变过程看成是双分

引用格式：任晓伟，周亮，田晓语，等. 利用瞬态苂光技术研究单重态激子裂变的分子间距依赖关系. 科学通报, 2016, 61: 3379-3387 Ren X W, Zhou L, Tian X Y, et al. Intermolecular distance dependence of singlet exciton fission by using transient fluorescence technique (in Chinese). Chin Sci Bull, 2016, 61: 3379-3387, doi: 10.1360/N972015-01158 
子间两次同时进行的电子转移过程, 电子转移的结 果使得原先一个分子上的单重态激子 $\mathrm{S}_{1}$ 演变为两个 分子上的三重态激子 $\mathrm{T}_{1}$. 另一种是从“激发态内转 换” 的角度理解 ${ }^{[13,14]}$, 即单重态激子 $\mathrm{S}_{1}$ 先经过一个快 速的内转换过程进人一个局域于单体分子上但却具 有多激子特征的单重态暗态, 然后再演变为两个单 体分子上的三重态激子 $\mathrm{T}_{1}$. 经过上述两种过程形成 的 $^{1}(\mathrm{TT})_{i}$ 态是两个仅有弱耦合的局域三重态, 它可通 过一个很小的扰动(如有机分子内的核磁场)而发生 快速的退相干, 最终完成两个三重态激子的分离. 实 际上, 上述两种解释都只是在一定实验结果基础上 提出的假设, 还需要通过更多的实验结果来加以检 验和完善.

在以往的工作中, 磁场效应曾经是研究激子裂 变过程广泛使用的手段 ${ }^{[4,8,15 ~ 18]}$. 由于外加磁场可以 对激子裂变的速率产生调制作用, 因而可以诱导多 种被测量, 如光致发光强度、电致发光强度、光电流 密度等发生明显改变. 在此前的工作中 ${ }^{[18]}$, 通过借 鉴已有的理论模型 ${ }^{[13]}$ 和实验结果 ${ }^{[6]}$, 本研究对由激 子裂变过程引起的材料光致发光的磁场效应进行了 分析, 尝试建立磁场效应幅度与激子裂变速率之间 的联系. 但在一般情况下, 磁场效应的幅度受多种因 素影响, 即使幅度较大也不能简单说明激子裂变的 速率快. 稳态下的磁场效应测量只能作为研究激子 裂变过程间接的、验证性的实验方法, 很难得到直接 的、定量的结果. 实验上对激子裂变过程直接的研究 主要是通过以下两种瞬态测量方法: (1) 是测量材料 光吸收的衰减过程 ${ }^{[6,18 ~ 23]}$, 吸收谱中不同的吸收波长 或吸收带对应着材料中不同的激发态, 单重态和三 重态激子的浓度变化反映在吸收谱的强度随时间的 变化上; (2) 是测量材料光发射的衰减过程 ${ }^{[7 ~ 9]}$, 材料 光致发光强度的变化只反映单重态激子的浓度变化. 对于测量结果的分析, 都是采用微分方程组对衰减 曲线进行数值拟合, 通过理论曲线与实验结果的对 比来推断激子裂变过程中各个不同激发态间的转化 过程及其速率.

本实验中, 利用双源共蒸发方法将具有激子裂 变特性的红苂烯(rubrene)分子掺杂到常用的有机材 料三 (8-差基喹啉)铝 $\left(\mathrm{Alq}_{3}\right)$ 中, 形成非晶态的 $\mathrm{Alq}_{3}$ : rubrene掺杂薄膜. 假设 rubrene分子可以均匀地分布 于混合膜中, 则通过设定 rubrene分子的掺杂浓度, 就可以控制相邻rubrene分子之间的平均间距, 改变
激子裂变与激子聚变过程的速率. 对不同掺杂浓度 的样品在室温下测量了光致发光的瞬态衰减过程, 用耦合的微分方程组描述了在激子裂变过程中各激 发态的动态变化, 来分析 rubrene分子间的激子裂变 过程, 拟合结果与实验曲线取得了很好的一致. 实验 发现, 激子裂变过程中的速率 $k_{-2}$ 随rubrene分子平均 间距的增大呈指数下降规律，这与有机材料中常见 的Dexter能量转移(Dexter energy transfer, DT)过程非 常相似 ${ }^{[24,25]}$. 对比 SF的双电子转移过程与 DT的双电 子转移过程, 可看出二者有明显的相似性, 这从实验 角度证明 SF过程的双电子转移模型是合理的, 研究 结果可以认为是对双电子转移模型的有力支持.

\section{1 掺杂薄膜的制备与测量}

本实验中, 在高真空 $\left(\sim 10^{-5} \mathrm{~Pa}\right)$ 条件下通过双源 共蒸发方法, 将 rubrene和 $\mathrm{Alq}_{3}$ 两种材料以不同的质 量比进行掺杂, 形成 $\mathrm{Alq}_{3}$ : $\operatorname{rubrene}(x \%)$ 混合薄膜. 在 制备样品时, 通过控制两个蒸发源各自的蒸发速率, 可以比较精确地控制 rubrene分子掺杂的质量百分比 $x \%$. 实验中, $x$ 的值被分别设定为 $50,40,30,20,14,2$, 以获得不同的激子裂变速率. 在蒸发过程中, 使用的 石英玻璃衬底始终保持在室温, 因此制备的薄膜样 品均为非晶态结构. 使用Edinburgh FLS-920稳态/瞬 态苂光光谱仪, 在室温下测量了全部样品的光致发 光谱及其瞬态衰减过程, 光致发光的激发波长为 355 $\mathrm{nm}$. 有关样品的制备与测量, 在此前的工作中已有 一些介绍 ${ }^{[18]}$, 故不赘述. 此外, 为了验证掺杂的 rubrene分子是否发生了分子间的聚集, 还对样品的 光致发光谱进行了变温测量.

\section{2 样品的光致发光谱及其瞬态衰减曲线}

图1展示了在不同的掺杂浓度下混合薄膜的光致 发光谱, 画图时将所有的谱线都进行了归一化处理, 图1的插图展示了 $\mathrm{Alq}_{3}$ 分子发光与 rubrene分子发光之 间的对比. 室温下, $\mathrm{Alq}_{3}$ 光谱的峰位波长约为 $525 \mathrm{~nm}$, 而 rubrene光谱的峰位波长约为 $562 \mathrm{~nm}$, 二者有明显 的差别. 由图1可见, 混合薄膜的发光全部来源于掺 杂的rubrene分子, 没有来自于 $\mathrm{Alq}_{3}$ 分子的发光, 其主 发光峰的中心波长和形状都非常一致, 所有谱线之 间的差别都很小.

在稳态下, 光致发光的强度 $F_{\mathrm{PL}}$ 近似有如下的 关系: 


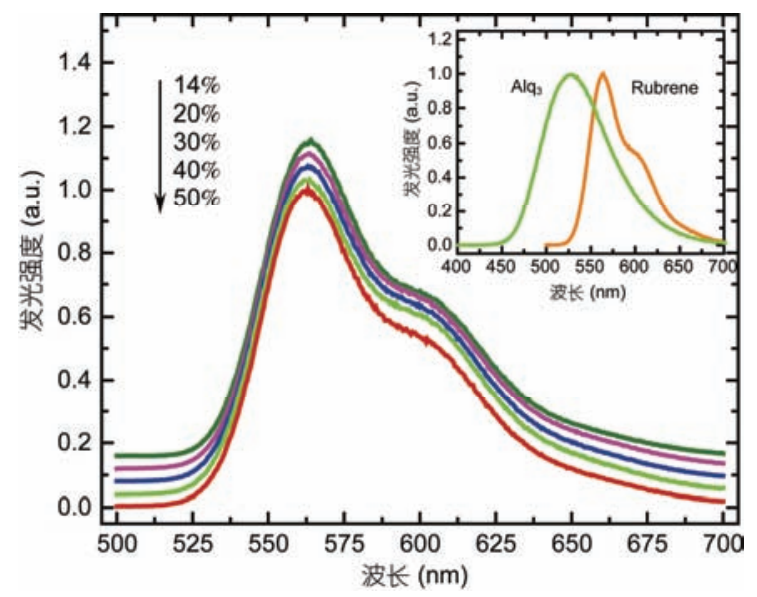

图 1 (网络版彩色)不同掺杂浓度下 $\mathrm{Alq}_{3}$ : rubrene薄膜的发光谱, 插 图显示 $\mathrm{Alq}_{3}$ 发光与rubrene发光之间的对比

Figure 1 (Color online) Spectra of $\mathrm{Alq}_{3}$ : rubrene films with different doping concentrations. The insert shows the comparison of fluorescence from $\mathrm{Alq}_{3}$ and rubrene

$$
F_{\mathrm{PL}} \propto k_{\mathrm{S}} /\left(k_{\mathrm{S}}+k_{\mathrm{SF}}\right) .
$$

其中, $k_{\mathrm{S}}$ 表示 $\mathrm{S}_{1}$ 态激子的发光速率, $k_{\mathrm{SF}}$ 表示激子裂变 过程(即 $\mathrm{S}_{1}+\mathrm{S}_{0} \rightarrow \mathrm{T}_{1}+\mathrm{T}_{1}$ 的整个过程)的表观速率. 根据 Johnson-Merrifield(J-M) 唯象理论 ${ }^{[2]}, k_{\mathrm{SF}}$ 可表示为 $k_{\mathrm{SF}}=k_{-2} \sum_{i=1}^{9}\left|C_{\mathrm{S}}^{i}\right|^{2} /\left(1+k_{2}\left|C_{\mathrm{S}}^{i}\right|^{2} / k_{-1}\right)$, 其中 $\left|C_{\mathrm{S}}^{i}\right|^{2}$ 表示每 个 $(\mathrm{TT})_{i}$ 态中所包含单重态的概率幅. 对 rubrene分子, 由于 $E\left(\mathrm{~S}_{1}\right)<2 E\left(\mathrm{~T}_{1}\right)$, 可知其激子裂变过程是一个热激 发的过程. 当温度降低时, 由于速率 $k_{\mathrm{SF}}$ 被抑制, 而速 率 $k_{\mathrm{S}}$ 不受温度影响, 则薄膜的光致发光强度 $F_{\mathrm{PL}}$ 将逐 渐增强. 图2描绘了 $\mathrm{Alq}_{3}$ : rubrene(50\%)混合薄膜在不 同温度下的发射光谱, 低温下发光明显增强, 在其他 样品中也观察到了同样的现象.

此外, 掺杂体系通常会存在一个重要的疑问, 就 是薄膜中的掺杂分子是否会发生某种方式的聚集. 是否在 $\mathrm{Alq}_{3}$ : rubrene掺杂薄膜中出现了 rubrene分子的 聚集, 可以通过不同温度下薄膜发光谱线的变化来 判断 ${ }^{[17]}$. 在图 2 的插图中, 对 $\mathrm{Alq}_{3}$ : rubrene (50\%)掺杂 薄膜在不同温度下的发光谱进行了归一化处理. 其 中波长在560 570 nm附近的发光峰(峰A), 对应着分 子从 $\mathrm{S}_{1}$ 态向 $\mathrm{S}_{0}$ 态的跃迁; 而波长在610 620 nm附近 的发光峰(峰B), 对应着分子从 $S_{1}$ 态向 $S_{0}$ 态的第一振 动能级的跃迁. 理论与实验已经证明, 这两个发光峰 之间高度的比值会受到分子聚集效应的影响 ${ }^{[26,27]}$. 但在插图中, 两个发光峰的相对高度并没有随着温 度的下降发生太明显的变化. 只是在低温下发光峰

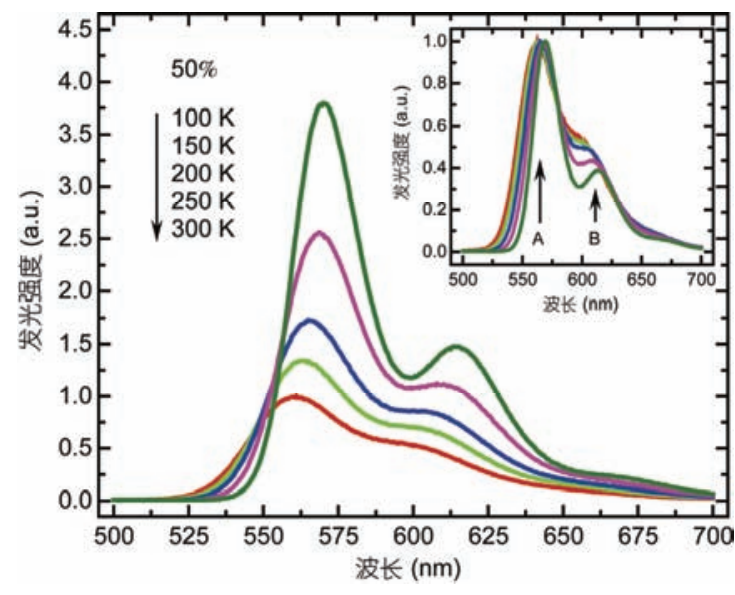

图 2 (网络版彩色)不同温度下 $\mathrm{Alq}_{3}$ : rubrene(50\%)薄膜的发光谱, 在 插图中对谱线进行了归一化处理

Figure 2 (Color online) Spectra of $\mathrm{Alq}_{3}$ : rubrene (50\%) films measured at different temperatures. All the curves are normalized in the insert

变得更尖锐且发生小幅度的红移, 这些变化是由其 他因素引起的，与分子间的聚集效应无关. 因此，可 以推断掺杂的rubrene分子大致均匀地分布在薄膜中, 没有发生明显的聚集.

由图1可见，室温下 rubrene分子发光的峰值约在 $562 \mathrm{~nm}$ 处. 在FLS-920苂光光谱仪中, 利用单色仪将 波长为562 $\mathrm{nm}$ 的发光选出, 在0 200 ns的范围内测量 了发光的瞬态衰减过程. 测量结果如图3所示. 比较 不同掺杂浓度样品的发光衰减曲线, 可看出在衰减 过程刚开始约 $20 \mathrm{~ns}$ 的时间范围内, rubrene的掺杂浓 度越高, 发光的衰减速度越快, 表明该样品中的激子 裂变速率越快. 而在大约60 ns之后, 发光的衰减速 度明显变慢. 因为此时样品中的 $\mathrm{S}_{1}$ 态激子已基本衰 减完毕, 但仍存在大量通过激子裂变过程产生的长 寿命的 $\mathrm{T}_{1}$ 态激子, 这些 $\mathrm{T}_{1}$ 态激子在进行非辐射复合 的同时, 又通过激子聚变过程不断地产生新的 $\mathrm{S}_{1}$ 态 激子, 进而产生发光. 通常在苂光材料中 $\mathrm{T}_{1}$ 态激子的 寿命很长, 故而这部分发光的衰减速度比较缓慢. 特 别的, 图 3 的插图展示了测量仪器的响应函数, 即 $\mathrm{IRF}$ (instrumental response function)曲线. 该曲线的半 高宽度约为 $0.63 \mathrm{~ns}$, 远小于混合薄膜中 $\mathrm{S}_{1}$ 态激子的平 均寿命. 插图中IRF曲线与 $50 \%$ 掺杂样品发光衰减曲 线的对比, 表明测量仪器的时间分辨能力对测量结 果没有影响.

为了对样品的发光衰减曲线进行拟合, 需要知 道rubrene分子中 $\mathrm{S}_{1}$ 态激子的发光速率 $k_{\mathrm{S}}$, 为此特别制 备了 $\mathrm{Alq}_{3}$ : rubrene(2\%)混合薄膜并进行了瞬态测量, 


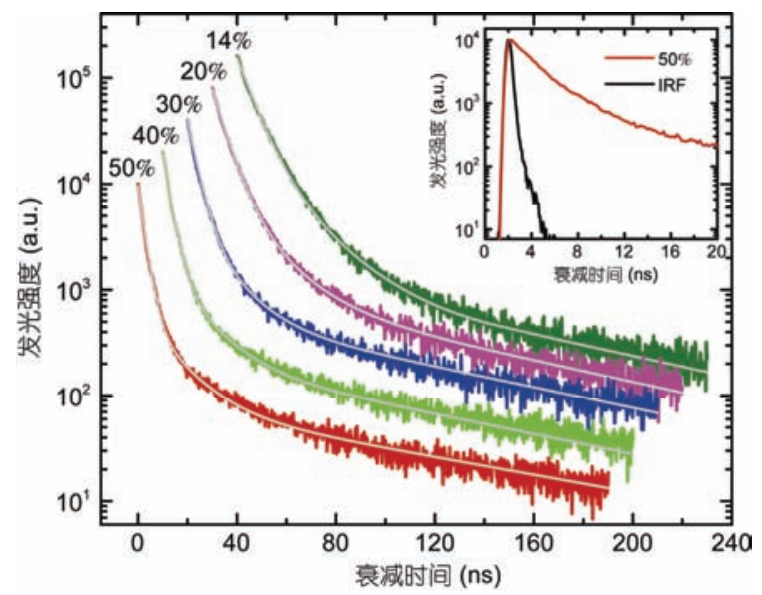

图 3 (网络版彩色) 不同掺杂浓度下混合薄膜发光的瞬态衰减曲线和 拟合曲线, 插图显示了仪器响应函数曲线

Figure 3 (Color online) Time-resolved fluorescence decay of mixed films with different doping concentrations. The insert shows the curve of instrumental response function (IRF)

测量结果展示在图4中. 如图4所示, 当rubrene分子 的掺杂浓度仅为 $2 \%$ 时, 样品光致发光的衰减近乎呈 指数下降规律. 因为掺杂的 rubrene分子之间平均距 离太大, 近于孤立的 rubrene分子既不能发生 $\mathrm{S}_{1}$ 态激 子的裂变也没有 $\mathrm{T}_{1}$ 态激子的聚变, 光照激发的 $\mathrm{S}_{1}$ 态 激子都是通过辐射复合的方式退激. 在发光开始衰 减的最初约 $30 \mathrm{~ns}$ 的时间范围内, 可以认为发光强度 的下降严格正比于指数 $\exp \left[-k_{\mathrm{S}} t\right]$, 因此根据这一段发 光的下降速度即可确定 rubrene分子中 $\mathrm{S}_{1}$ 态激子的发 光速率 $k_{\mathrm{S}}=0.120 \mathrm{~ns}^{-1}$, 对应的 $\mathrm{S}_{1}$ 态激子的平均寿命约

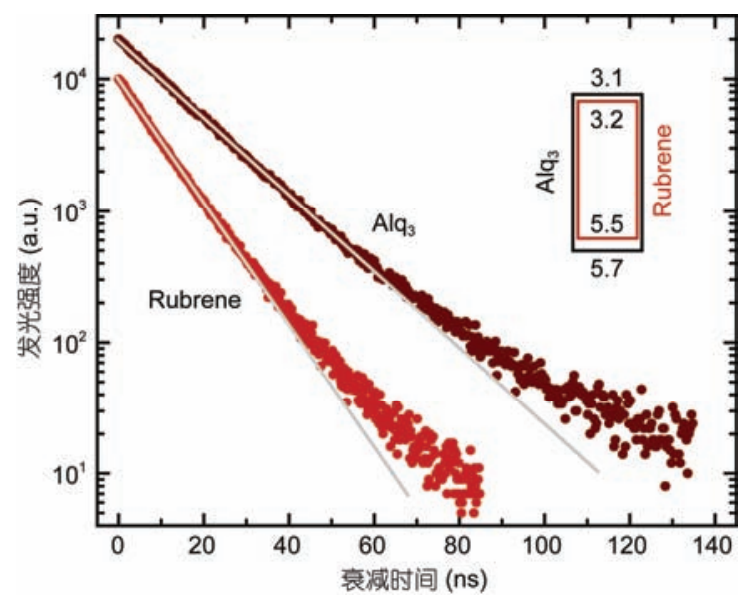

图 4 (网络版彩色) rubrene分子和 $\mathrm{Alq}_{3}$ 分子中 $\mathrm{S}_{1}$ 态激子平均寿命的测 量结果, 插图中显示了两种分子的能级结构

Figure 4 (Color online) The measurement results of average lifetime of $\mathrm{S}_{1}$ excitons in rubrene and $\mathrm{Alq}_{3}$ molecules. The insert shows the energy levels of these two molecules
为 $8.3 \mathrm{~ns}$. 随着 rubrene分子的掺杂浓度逐渐增大, 相 邻rubrene分子间的平均距离逐渐减小，激子裂变过 程随之逐渐增强, 使得光致发光强度在初始阶段的 衰减越来越快。实验发现，在 rubrene的掺杂浓度超 过 $10 \%$ 之后, 混合薄膜中的激子裂变过程已经非常 明显. 作为参考比较, 图4也给出了纯 $\mathrm{Alq}_{3}$ 薄膜发光 衰减的测量结果. 根据最初 $70 \mathrm{~ns}$ 时间范围内的发光 衰减, 可确定 $\mathrm{Alq}_{3}$ 分子中 $\mathrm{S}_{1}$ 态激子的平均寿命约为 $15 \mathrm{~ns}$, 这与此前文献报道的结果基本一致 ${ }^{[28]}$.

虽然目前普遍认同用公式(1)来描述激子裂变的 动力学过程, 但在具体分析发光的瞬态衰减时还存 在不同看法. 动力学过程所涉及的主要问题, 是受激 发的分子在公式(1)所包含的 3 个状态之间如何实际 演化? 不同的研究者对此提出了各自的看法, 其中 的疑问主要有以下两点: (1) 在 $i$ 不同的 $(\mathrm{TT}){ }_{i}$ 态 $(i=$ 1 9)之间有可能由于自旋-晶格弛豫作用引起相互间 的转化 ${ }^{[8,15]}$, 使得原先不具有单重态特征的 ${ }^{S}(\mathrm{TT})_{i}$ 态 ( $S=5$ 为五重态, $S=3$ 为三重态)也可演化为具有单态特 征的 ${ }^{1}(\mathrm{TT})_{i}$ 态, 而原先具有单态特征的 ${ }^{1}(\mathrm{TT})_{i}$ 态也可 演化为不具有单重态特征的 ${ }^{S}(\mathrm{TT})_{i}$ 态, 因此自旋-晶格 弛豫作用对于激子裂变过程究竟会产生什么样的影 响?（2）由某一次激子裂变过程产生的一对孪生的 $\mathrm{T}_{1}$ 态激子, 是否会与其他的激子裂变过程所产生的 $\mathrm{T}_{1}$ 态激子发生相互作用, 进而再次发生激子聚变过 程 ${ }^{[29]}$ ? 上述的两个问题, 是对瞬态衰减过程进行曲 线拟合时必须要考虑的关键因素.

\section{3 瞬态衰减的动力学过程拟合}

如前所述, 激子裂变的动力学过程通常可用公 式(1)来进行描述. 在此基础上, 许多研究者试图采 用耦合的微分方程组(也称速率方程组)来拟合光致 发光的瞬态衰减过程, 然而拟合结果与测量曲线之 间大都有明显偏差. 在比较简化的情况下, 也可以采 用形式为 $A_{1} \mathrm{e}^{-t / \tau_{1}}+A_{2} \mathrm{e}^{-t / \tau_{2}}+A_{3} \mathrm{e}^{-t / \tau_{3}}$ 的近似公式拟合 发光的衰减, 虽然也可以得到较好的符合结果, 但公 式的物理意义不明确. 实际上, 采用耦合的微分方程 组来拟合发光的衰减过程是比较合理的方法, 但每 个微分方程的设定则与前述提到的两个问题密切相 关, 以下将分别对这两个问题进行详细讨论.

(1) 不同 ${ }^{S}(\mathrm{TT})_{i}$ 态之间发生自旋-晶格弛豫过程的 结果是什么? 根据J-M唯象理论 ${ }^{[2]}, \mathrm{S}_{1}+\mathrm{S}_{0}$ 态只能与具 有单重态特征的 ${ }^{1}(\mathrm{TT})_{i}$ 态之间发生相互转变, 而根据 
总自旋守恒的要求, 在 9 个 $(\mathrm{TT})_{i}$ 态中, 只有部分 $(\mathrm{TT})_{i}$ 态具有单重态特征, 即 $\mathrm{S}_{1}+\mathrm{S}_{0}$ 态只能与这几个 $(\mathrm{TT})_{i}$ 态 之间发生激子裂变和激子聚变过程. 而其他不具有单 重态特征的 ${ }^{S}(\mathrm{TT}){ }_{i}$ 态, 则可以通过自旋-晶格弛豫作用 转变为具有单重态特征的 ${ }^{1}(\mathrm{TT})_{i}$ 态 $^{[8,15]}$, 这实际上会增 加激子裂变和激子聚变的速率. (2) 由不同的激子裂 变事件所产生的 $\mathrm{T}_{1}$ 态激子之间是否可以发生 $\mathrm{T}_{1}$ 态激子 的聚变过程? 由于 $\mathrm{T}_{1}$ 态激子的寿命很长, 在载流子迁 移率较高的材料中 $\mathrm{T}_{1}$ 态激子的扩散距离较远, 由不同 的激子裂变过程产生的 $\mathrm{T}_{1}$ 态激子可以再次发生碰撞并 形成 ${ }^{1}(\mathrm{TT})_{i}$ 态, 进而发生激子聚变过程. 而在掺杂样品 中, rubrene分子之间被 $\mathrm{Alq}_{3}$ 分子间隔, 薄膜的载流子 迁移率较低, 由激子裂变过程形成的 $\mathrm{T}_{1}$ 态激子很难自 由移动. 因此, 可以认为 $\mathrm{T}_{1}$ 态激子的聚变过程, 只能 在由激子裂变过程产生的一对 $\mathrm{T}_{1}$ 态激子之间发生, 而 在由不同的裂变事件产生的不同的 $\mathrm{T}_{1}$ 态激子之间, 不 会发生再次的激子聚变过程 ${ }^{[7 \sim 9]}$.

根据上述讨论, 令 $N_{\mathrm{S}}$ 为 $\mathrm{S}_{1}$ 态激子的数量, $N_{\mathrm{D}}$ 为中 间过渡态 $\left.{ }^{1}(\mathrm{TT})\right)_{i}$ 态的数量, $N_{\mathrm{T}}$ 为 $\left(\mathrm{T}_{1}+\mathrm{T}_{1}\right)$ 态激子对的数 量, 可用一组耦合的微分方程来描述这 3 个状态的变 化, 具体形式如下:

$$
\begin{gathered}
\frac{\mathrm{d} N_{\mathrm{S}}}{\mathrm{d} t}=-\left(k_{\mathrm{S}}+k_{-2}\right) N_{\mathrm{S}}+k_{2} N_{\mathrm{D}}, \\
\frac{\mathrm{d} N_{\mathrm{D}}}{\mathrm{d} t}=-\left(k_{2}+k_{-1}\right) N_{\mathrm{D}}+k_{-2} N_{\mathrm{S}}+k_{1} N_{\mathrm{T}}, \\
\frac{\mathrm{d} N_{\mathrm{T}}}{\mathrm{d} t}=-\left(k_{1}+k_{\mathrm{T}}\right) N_{\mathrm{T}}+k_{-1} N_{\mathrm{D}} .
\end{gathered}
$$

其中, $k_{\mathrm{T}}$ 为 $\mathrm{T}_{1}$ 态激子发生非辐射复合的速率, 其他速 率前面已有说明。在公式 (3a), (3b), (3c) 中, 有 $k_{\mathrm{S}}\left(0.120 \mathrm{~ns}^{-1}\right), k_{-2}, k_{2}, k_{-1}, k_{1}, k_{\mathrm{T}}$ 共6个速率, 以及 $N_{\mathrm{S}}(0), N_{\mathrm{D}}(0), N_{\mathrm{T}}(0)$ 共 3 个初始值. 选择合理的数值并 进行迭代运算, 即可利用公式算出各个时刻 $N_{\mathrm{S}}(t)$, $N_{\mathrm{D}}(t)$ 和 $N_{\mathrm{T}}(t)$ 的值. 表 1 列出了发光衰减曲线的拟合参 数. 样品的光致发光强度正比于 $N_{\mathrm{S}}(t)$, 因此通过 $N_{\mathrm{S}}(t)$ 的变化即可得到光致发光的瞬态衰减过程, 曲线拟 合的结果显示在图3中. 由图3可见, 拟合曲线与实验 曲线符合得非常好, 说明对前述两个问题的处理和 3 个微分方程的设定都是非常合理的.

由表1可见, $\left(k_{\mathrm{S}}+k_{-2}\right),\left(k_{2}+k_{-1}\right),\left(k_{1}+k_{\mathrm{T}}\right) 3$ 者间的数 值差别较大, 且 $\left(k_{\mathrm{S}}+k_{-2}\right)>\left(k_{2}+k_{-1}\right)>\left(k_{1}+k_{\mathrm{T}}\right)$, 因此在最 简单的情况下, 可作如下近似, 即 $\mathrm{d} N_{\mathrm{S}} / \mathrm{d} t \approx-\left(k_{\mathrm{S}}+\right.$ $\left.k_{-2}\right) N_{\mathrm{S}}, \mathrm{d} N_{\mathrm{D}} / \mathrm{d} t \approx-\left(k_{2}+k_{-1}\right) N_{\mathrm{D}}, \mathrm{d} N_{\mathrm{T}} / \mathrm{d} t \approx-\left(k_{1}+k_{\mathrm{T}}\right) N_{\mathrm{T}}$. 它
表 1 图 3 中发光衰减曲线的拟合参数

Table 1 Fitting parameters used for simulated curves in Figure 3

\begin{tabular}{cccccc}
\hline 浓度 & $k_{-2}\left(\mathrm{~ns}^{-1}\right)$ & $k_{2}\left(\mathrm{~ns}^{-1}\right)$ & $k_{-1}\left(\mathrm{~ns}^{-1}\right)$ & $k_{1}\left(\mathrm{~ns}^{-1}\right)$ & $k_{\mathrm{T}}\left(\mathrm{ns}^{-1}\right)$ \\
\hline $50 \%$ & 0.253 & 0.025 & 0.062 & 0.011 & 0.010 \\
$40 \%$ & 0.197 & 0.029 & 0.062 & 0.012 & 0.011 \\
$30 \%$ & 0.122 & 0.034 & 0.062 & 0.013 & 0.012 \\
$20 \%$ & 0.058 & 0.051 & 0.062 & 0.013 & 0.012 \\
$14 \%$ & 0.032 & 0.102 & 0.062 & 0.013 & 0.012 \\
\hline
\end{tabular}

们的解是 3 个简单的指数下降函数, 这就是光致发光 的瞬态衰减曲线可以近似用公式 $A_{1} \mathrm{e}^{-t / \tau_{1}}+A_{2} \mathrm{e}^{-t / \tau_{2}}+$ $A_{3} \mathrm{e}^{-t / \tau_{3}}$ 来进行拟合的原因. 此外, ${ }^{1}(\mathrm{TT})_{i}$ 态实际上也 存在衰减过程, 其衰减速率应当与 $\mathrm{T}_{1}$ 态激子的衰减 速率处于同一量级, 但数值应有不同. 对这一问题的 处理, 是在方程 $3(\mathrm{~b})$ 和 (c)中将 ${ }^{1}(\mathrm{TT})_{i}$ 态自身的消灭归 并到 ${ }^{1}(\mathrm{TT})_{i}$ 态分解为 $\mathrm{T}_{1}+\mathrm{T}_{1}$ 态而后 $\mathrm{T}_{1}+\mathrm{T}_{1}$ 态自然衰减的 过程中, 这样就可以不用考虑 ${ }^{1}(\mathrm{TT})_{i}$ 态自身的衰减速 率, 并使微分方程得到简化.

\section{4 激子裂变过程的双电子转移模型分析}

如图 4的插图所示, $\mathrm{Alq}_{3}$ 与 rubrene两种分子的 HOMO(highest occupied molecular orbit)能级(5.7与 $5.5 \mathrm{eV}$ )和LUMO(lowest unoccupied molecular orbit)能 级 $\left(3.1\right.$ 与 $3.2 \mathrm{eV}$ ) 的差别都不大. 在混合薄膜中, $\mathrm{Alq}_{3}$ 分子对 rubrene分子起到间隔作用，使 rubrene分子在 不同的掺杂浓度下获得不同的分子间平均距离. 假 设掺杂的 rubrene分子在混合薄膜中的分布是均匀的, 则可以根据这两种材料各自的摩尔质量与摩尔体积, 将薄膜中rubrene分子的平均间距 $d$ 大致算出来. 根据 Aldrich公司给出的数据, 处于非晶态时rubrene的摩尔 质量是 $532.67 \mathrm{~g}$, 摩尔体积是 $452.9 \mathrm{~cm}^{3}$. 因此薄膜中 每个 rubrene分子占据的体积约为 $0.75 \mathrm{~nm}^{3}$. 同样, 处 于非晶态时 $\mathrm{Alq}_{3}$ 的摩尔质量为 $459.43 \mathrm{~g}$, 摩尔体积为 $350.7 \mathrm{~cm}^{3}$, 因此薄膜中每个 $\mathrm{Alq}_{3}$ 分子占据的体积约 为 $0.58 \mathrm{~nm}^{3}$. 由于有 $\mathrm{Alq}_{3}$ 分子的间隔作用, 在混合膜 中 rubrene分子的掺杂浓度越低，相邻的rubrene分子 间的平均距离 $d$ 越大, 因而 rubrene分子间发生激子裂 变的速率越低.

图5显示了表1中所列速率 $k_{-2}$ 的值随不同掺杂浓 度所对应的分子间距 $d$ 的变化, 实验数据基本符合 $k_{-2}$ $\propto \exp (-d / D)$ 的变化趋势, 其中 $D \approx 0.28 \mathrm{~nm}$ 为拟合参 数. 这种反应速率与分子间距的指数下降关系, 与有 


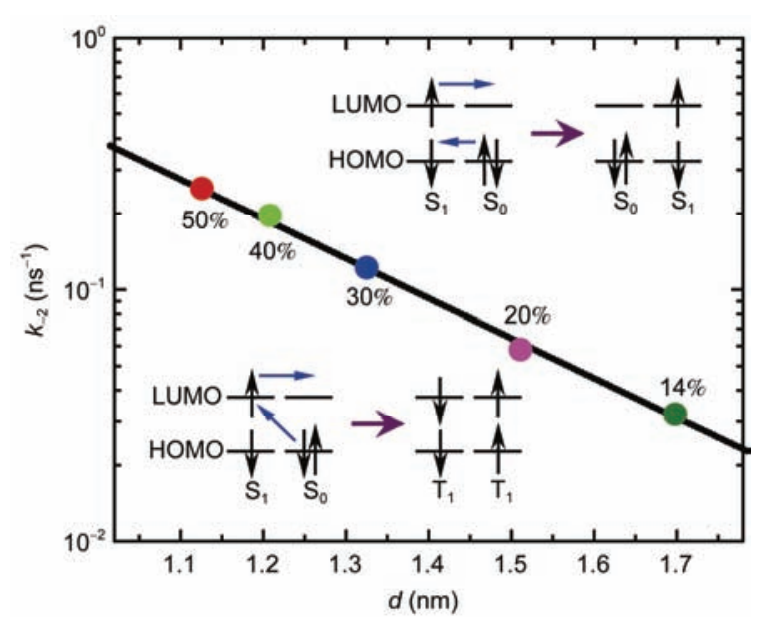

图 5 (网络版彩色) 速率 $k_{-2}$ 与分子间距 $d$ 的指数关系, 插图显示 $\mathrm{DT}$ (右上)与 $\mathrm{SF}$ (左下)过程的双电子转移模型

Figure 5 (Color online) The exponential relationship between rate $k_{-2}$ and intermolecular distance $d$. The double-electron transfer models of DT (upper right) and SF (lower left) processes are shown as insert

机材料中常见的Dexter能量转移过程非常相似. 在一 定范围内, $\mathrm{DT}$ 过程的速率 $k_{\mathrm{DT}}$ 与分子间距 $d_{\mathrm{DA}}$ 的关系 为 ${ }^{[24,25]}$

$$
k_{\mathrm{DT}}=K J_{\mathrm{DA}} \exp \left(-2 d_{\mathrm{DA}} / L\right) .
$$

上式中的 $J_{\mathrm{DA}}$ 表示 DT过程中给体(donor, 用D表示)的 发射光谱与受体(accepter, 用 $\mathrm{A}$ 表示)的吸收光谱之间 的重叠积分, $d_{\mathrm{DA}}$ 表示给体分子与受体分子间的距离, $L$ 是两种分子的范德瓦尔斯半径之和. DT过程的发生 需要两个分子相关轨道的电子云有一定的相互交叠, 以保证分子间有足够的耦合强度, 上式中的因子 $K$ 即 是与分子交叠情况有关的常数. 由于分子上电子云 的密度随离开分子的距离指数衰减, 如果不考虑两 个分子上电子云交叠的细节, 则发生DT过程的速率 与两个分子之间的距离满足(4)式的关系. 分子间DT 过程的作用距离较小, 通常在 $15 \AA$ 的范围内. 由 图5可见, $\mathrm{SF}$ 过程的作用距离大致在 $20 \AA$ 的范围以 内, 两者相差不大.

当两个有机分子间发生DT过程时, 是以载流子 直接交换的方式在分子间传递能量. 当一个处于激 发态的分子和另一个处于基态的分子距离很近, 以 至于电子云彼此交叠的时候, 处于激发态的分子上 的电子就能直接迁移到邻近的基态分子上, 在完成 电荷转移的同时完成能量的迁移. 如图5右上角的插 图所示, DT过程通常可看作是通过两个电子在给体 分子和受体分子的HOMO和LUMO能级上同时发生
电子转移而完成的 ${ }^{[24]}$. 图5所描述的, 是一个从单重 态到单重态的DT过程，而从三重态到三重态的DT过 程与此类似. 与DT过程进行对比, SF过程的双电子 转移模型的物理过程十分相似. 如图5左下角的插图 所示, SF过程也包括两次HOMO和LUMO能级上同时 发生的电子转移, 在完成电荷转移的同时完成能量 的迁移 ${ }^{[2,10,11]}$. 不同的是, 为了实现另一个基态分子 的激发, 需要原先基态分子 $\mathrm{HOMO}$ 能级上的电子转 移到原先激发态分子的LUMO能级上去, 以实现激 子能量的分配. 但不管是DT过程还是 SF过程, 都满 足总自旋守恒这一基本要求. 此前, 普遍认为 $\mathrm{SF}$ 过程 中的两次电子转移既可以同时发生也可以先后发生, 其先后次序可以是随机的. 而最近的研究结果表 明 $^{[30]}$, 这两次电子转移过程必须同步发生, 否则就不 能保证SF过程发生前后激发态分子的振动相干性. 因 此, 双电子转移模型也被称之为“超交换模型”, [11].

\section{5 总结}

本实验中, 将具有激子裂变特性的 rubrene分子 以不同的浓度掺杂于 $\mathrm{Alq}_{3}$ 有机薄膜中, 通过调控 rubrene分子的掺杂浓度来改变薄膜中相邻rubrene分 子的平均间距，这将直接影响相邻分子上电子云交叠 的程度, 从而改变相邻rubrene分子间发生激子裂变过 程的速率. 在室温下测量了具有不同rubrene掺杂浓度 的有机薄膜光致发光的瞬态衰减过程. 理论上, 基于 三状态反应模型，可以采用一组耦合的微分方程来描 述激子裂变过程中各个激发态的数量随时间的变化. 利用合理的微分方程组对发光的瞬态衰减曲线进行了 拟合, 理论曲线与实验曲线取得了很好的符合. 最重 要的是, 数据分析发现激子演变速率随分子间距的增 大呈指数下降规律, 这与有机材料中常见的Dexter能 量转移过程非常相似. 函数关系上的相似性来源于二 者物理图像上的相似性, 这两种过程都可以通过双电 子转移模型来理解, 这从实验角度证明了激子裂变的 双电子转移模型的合理性. 采用这样的思路, 有望通 过更多的实验研究分析出影响激子裂变过程速率的各 个重要物理因素, 最终弄清激子裂变过程的物理机制, 为设计和合成新的具有激子裂变特性的有机分子提供 理论上的指导. 此外, 对激子裂变动力学过程的研究, 也有助于分析激子裂变过程在有机光伏器件中的微观 图像, 为有效利用激子裂变过程提高器件的量子效率 提供理论与实验帮助. 


\section{参考文献}

1 Paci I, Johnson C J, Chen X D, et al. Singlet fission for dye-sensitized solar cells: Can a suitable sensitizer be found? J Am Chem Soc, 2006, 128: 16546-16553

2 Smith M B, Michl J. Singlet fission. Chem Rev, 2010, 110: 6891-6936

3 Reusswig P D, Congreve D N, Thompson N J, et al. Enhanced external quantum efficiency in an organic photovoltaic cell via singlet fission exciton sensitizer. Appl Phys Lett, 2012, 101: 113304

4 Congreve D N, Lee J, Thompson N J, et al. External quantum efficiency above $100 \%$ in a singlet-exciton-fission-based organic photovoltaic cell. Science, 2013, 340: 334-337

5 Wu T C, Thompson N J, Congreve D N, et al. Singlet fission efficiency in tetracene-based organic solar cells. Appl Phys Lett, 2014, 104: 193901

6 Ma L, Zhang K K, Kloc C, et al. Singlet fission in rubrene single crystal: Direct observation by femtosecond pump-probe spectroscopy. Phys Chem Chem Phys, 2012, 14: 8307-8312

7 Jankus V, Snedden E W, Bright D W, et al. Competition between polaron pair formation and singlet fission observed in amorphous rubrene films. Phys Rev B, 2013, 87: 224202

8 Piland G B, Burdett J J, Kurunthu D, et al. Magnetic field effects on singlet fission and fluorescence decay dynamics in amorphous rubrene. J Phys Chem C, 2013, 117: 1224-1236

9 Li J, Chen Z H, Zhang Q M, et al. Temperature-dependent singlet exciton fission observed in amorphous rubrene films. Org Electron, 2015, 26: 213-217

10 Greyson E C, Vura-Weis J, Michl J, et al. Maximizing singlet fission in organic dimers: Theoretical investigation of triplet yield in the regime of localized excitation and fast coherent electron transfer. J Phys Chem B, 2010, 114: 14168-14177

11 Berkelbach T C, Hybertsen M S, Reichman D R. Microscopic theory of singlet exciton fission. II. Application to pentacene dimers and the role of superexchange. J Chem Phys, 2013, 138: 114103

12 Yost S R, Lee J, Wilson M W B, et al. A transferable model for singlet-fission kinetics. Nat Chem, 2014, 6: 492-497

13 Zimmerman P M, Zhang Z Y, Musgrave C B. Singlet fission in pentacene through multi-exciton quantum states. Nat Chem, 2010, 2: 648-652

14 Zimmerman P M, Bell F, Casanova D, et al. Mechanism for singlet fission in pentacene and tetracene: From single exciton to two triplets. J Am Chem Soc, 2011, 133: 19944-19952

15 Tarasov V V, Zoriniants G E, Shushin A I, et al. The role of spin-lattice relaxation in magnetic field effects on the luminescence of amorphous and polycrystalline rubrene films. Chem Phys Lett, 1997, 267: 58-64

16 Zhang Y, Lei Y L, Zhang Q M, et al. Thermally activated singlet exciton fission observed in rubrene doped organic films. Org Electron, 2014, 15: 577-581

17 Li J, Chen Z H, Lei Y L, et al. Competition between singlet exciton fission, radiation, and dissociation measured in rubrene-doped amorphous films. Syn Metal, 2015, 207: 13-17

18 Chen Z H, Li J, Xiong Z H, et al. Singlet exciton fission process in rubrene-doped organic films (in Chinese). Chin Sci Bull, 2014, 59: 1942-1948 [陈中海, 李婧, 熊祖洪, 等. 红苂烯掺杂有机薄膜中的单重态激子裂变过程. 科学通报, 2014, 59: 1942-1948]

19 Roberts S T, McAnally R E, Mastron J N, et al. Efficient singlet fission discovered in a disordered acene film. J Am Chem Soc, 2012, 134: 6388-6400

20 Musser A J, Al-Hashimi M, Maiuri M, et al. Activated singlet exciton fission in a semiconducting polymer. J Am Chem Soc, 2013, 135: $12747-12754$

21 Wilson M W B, Rao A, Johnson K, et al. Temperature-independent singlet exciton fission in tetracene. J Am Chem Soc, 2013, 135: 16680-16688

22 Pensack R D, Tilley A J, Parkin S R, et al. Exciton delocalization drives rapid singlet fission in nanoparticles of acene derivatives. J Am Chem Soc, 2015, 137: 6790-6803

23 Zhang B, Zhang C F, Wang R, et al. Nonlinear density dependence of singlet fission rate in tetracene films. J Phys Chem Lett, 2014, 5: 3462-3467

24 Albinsson B, Eng M P, Pettersson K, et al. Electron and energy transfer in donor-acceptor systems with conjugated molecular bridges. Phys Chem Chem Phys, 2007, 9: 5847-5864

25 Monguzzi A, Tubino R, Meinardi F. Upconversion-induced delayed fluorescence in multicomponent organic systems: Role of Dexter 
energy transfer. Phys Rev B, 2008, 77: 155122

26 Lim S H, Bjorklund T G, Spano F C, et al. Exciton delocalization and superradiance in tetracene thin films and nanoaggregates. Phy Rev Lett, 2004, 92: 107402

27 Clark J, Silva C, Friend R H, et al. Role of intermolecular coupling in the photophysics of disordered organic semiconductors: Aggregate emission in regioregular polythiophene. Phys Rev Lett, 2007, 98: 206406

28 Palilis L C, Melinger J S, Wolak M A, et al. Excitation energy transfer in tris(8-hydroxyquinolinato)aluminum doped with a pentacene derivative. J Phys Chem B, 2005, 109: 5456-5463

29 Bayliss S L, Chepelianskii A D, Sepe A, et al. Geminate and nongeminate recombination of triplet excitons formed by singlet fission. Phys Rev Lett, 2014, 112: 238701

30 Musser A J, Liebel M, Schnedermann C, et al. Evidence for conical intersection dynamics mediating ultrafast singlet exciton fission. Nat Phys, 2015, 11: 352-357 


\title{
Intermolecular distance dependence of singlet exciton fission by using transient fluorescence technique
}

\author{
REN XiaoWei, ZHOU Liang, TIAN XiaoYu \& ZHANG Yong \\ School of Physical Science and Technology, Southwest University, Chongqing 400715, China
}

In rubrene-doped organic thin films, the average intermolecular distance between adjacent rubrene molecules was changed by controlling the doping concentration of rubrene. In rubrene: $\mathrm{Alq}_{3}$ composite films, the average distance of doped rubrene molecules was decreased from $\sim 1.7 \mathrm{~nm}$ down to $\sim 1.1 \mathrm{~nm}$ when the weight ratio of rubrene was increased from $14 \%$ to $50 \%$. The variation of intermolecular distance affected the magnitude of overlap of exciton wave function between adjacent rubrene molecules, hence tuning the strength of intermolecular coupling and leading to different rate of singlet exciton fission between neighboring rubrene molecules. In experiment, the steady-state photoluminescence spectra were firstly measure. And time-resolved fluorescence decay curves of different samples were recorded at room temperature by using transient fluorescence spectroscopy. All the excited fluorescence was from doped rubrene. In theory, based on a three-state reaction model of " $\mathrm{S}_{1}+\mathrm{S}_{0} \leftrightarrow{ }^{1}(\mathrm{TT})_{i} \leftrightarrow \mathrm{T}_{1}+\mathrm{T}_{1}$ ", the dynamic process of singlet exciton fission in amorphous rubrene was appropriately described by using three coupled rate equations. All the measured transient decay curves of photoluminescence of rubrene-doped films could be well fitted by the iterative calculation of rate equations. And a detailed analysis about the critical issues which were related with curve-fitting was given. According to the fitting parameters, it was found that the rate of " $\mathrm{S}_{1}+\mathrm{S}_{0} \rightarrow^{1}(\mathrm{TT})_{i}$ " process exponentially decreased with the increasing rubrene distance. Such a phenomenon was not reported before. This is very similar with the previous observations in the process of Dexter energy transfer in which the rate of interaction also exponentially decreased with the increasing donor-acceptor distance. In Dexter process, the energy transfer can be considered as the process of two electrons' hopping between donor and acceptor molecules. Similarly, singlet exciton fission process also involves two electrons' hopping between two adjacent rubrene molecules. According to this physical image, the excitation of triplet excitons and redistribution of energy of singlet exciton are accomplished by the formation of an intermediate triplet pair state. The enlarged intermolecular distance would result in exponentially decayed electron-transfer rates, thus decreasing the comprehensive rate constant of singlet fission. Since both of two processes can be explained by "double-electron transfer model", the similarity in functional relationship could actually be regarded as the first experimental confirmation of "double-electron transfer model" for singlet exciton fission. This is of significant value for clarifying the physical mechanism of singlet fission process. Furthermore, an in-depth investigation about such an exponential relationship between the exciton fission rate and intermolecular distance will be helpful for analyzing the dynamics and key factors of singlet exciton fission process.

rubrene, photoluminescence, singlet fission, electron transfer

doi: 10.1360/N972015-01158 